\title{
L'Assemblée des délégués de la FMH valide le concept détaillé
}

\author{
Kerstin Schutz ${ }^{a}$, Christian Oeschger ${ }^{b}$ \\ a Experte, division Médecine et tarifs ambulatoires; ${ }^{b}$ Collaborateur scientifique, division Médecine et tarifs ambulatoires
}

Lors de l'Assemblée des délégués (AD) de la FMH du 26 janvier 2017, les délégués des organisations faîtières, de l'AMDHS, de l'ASMAC et de femmes médecins suisse se sont notamment prononcés en faveur de la révision tarifaire TARCO. En acceptant le concept détaillé, les principes tarifaires et le concept de valeur intrinsèque, l'AD a donné le feu vert à la poursuite des travaux de révision.

Suite à la séance de l'Assemblée des délégués $(\mathrm{AD})$ de la FMH du 7 septembre 2016, les collaborateurs de la division Médecine et tarifs ambulatoires ont travaillé activement à l'élaboration du concept détaillé pour le projet TARCO. Ce concept a été présenté aux délégués de l'AD le 26 janvier 2017 avec les principes tarifaires, le concept de valeur intrinsèque et plusieurs autres documents complémentaires pour approbation. Les délégués ont suivi la proposition de la FMH et chargé ainsi le département Médecine et tarifs ambulatoires de poursuivre les travaux.

\section{Changements fondamentaux des principes tarifaires}

Outre le concept détaillé, les délégués ont dû se prononcer sur les principes tarifaires remaniés. A cet égard, il est important de relever que la FMH ne poursuivra pas la neutralité des coûts, qu'elle ne normalisera pas la structure tarifaire et qu'elle se concentrera, dans le cadre du projet TARCO, sur le secteur ambulatoire (cabinets). Par ailleurs, le soutien médical et les prestations générales en salle d'opération seront intégralement revus et révisés d'après les retours du corps médical. Enfin, la plausibilité des prestations à l'acte présentant un volume important (par chapitre) devra être contrôlée comme cela a été demandé et, le cas échéant, certaines devront à nouveau être dotées d'une valeur intrinsèque qualitative.

\section{Accent sur les prestations ambulatoires (cabinets)}

Certains partenaires tarifaires ont décidé de développer leur propre proposition indépendamment de la
FMH et ont soumis au Conseil fédéral des propositions d'adaptation du tarif actuel en octobre 2016. La FMH ne dispose donc plus de toutes les données fournies par les partenaires de la révision lors du précédent projet, mais uniquement des données ambulatoires relatives aux cabinets, c'est pourquoi le département a proposé d'axer le projet TARCO principalement sur ce secteur. Cette situation ne signifie cependant en aucun cas que le catalogue de prestations sera réduit, ni que la FMH ne défend plus les intérêts des médecins hospitaliers.

1. Toutes les prestations fournies en libre pratique seront tarifées du point de vue du cabinet. D’après les premières estimations, la révision tarifaire TARCO VO.1 contient environ 250 prestations ambulatoires purement hospitalières, qui ne seront plus prises en compte dans la suite du projet TARCO étant donné que la FMH ne dispose plus des données nécessaires pour calculer la prestation technique (PT). Comme pour les prestations ambulatoires des cabinets, la FMH continuera à s'investir pour la prestation médicale (PM) des prestations ambulatoires hospitalières qui sont dotées du même revenu de référence.

2. Pour la FMH, toutes les prestations médicales doivent être indemnisées selon un principe identique - qu'elles soient fournies dans un cabinet libéral, à l'hôpital ou par un médecin agréé. L'indemnisation se fonde sur le revenu de référence et les facteurs de valeur intrinsèque. Au lieu d'une tarification séparée des prestations ambulatoires hospitalières, la FMH défend un revenu de référence identique dans tout le secteur ambulatoire des hôpitaux comme taux minimal pour l'activité médicale. 


\section{Contrôle de la plausibilité des prestations à l'acte}

Le contrôle de la plausibilité des minutages des prestations à l'acte présentant un volume important et la vérification de l'application correcte de prestations à l'acte ou au temps font partie des objectifs du projet TARCO. De nombreuses sociétés affiliées à la FMH de même que l'OFSP et les répondants des coûts exigent que la plausibilité du temps requis pour fournir les prestations à l'acte soit contrôlée.

Dans un premier temps, l'accent est mis sur le contrôle des minutages des prestations à l'acte présentant un volume important. Avec l'aide de la Haute école spécialisée bernoise (BFH), une étude scientifique est en cours de réalisation, laquelle servira également de concept pour la marche à suivre. La charge de travail devrait être à peu près la même pour toutes les sociétés de discipline concernées. La participation à ce projet est toutefois facultative pour les sociétés. La BFH est en train de peaufiner l'ébauche de concept pour ce projet. La décision concernant le concept définitif et la marche à suivre de la $\mathrm{BFH}$ pour le contrôle de la plausibilité sera prise par le groupe de pilotage TARCO («Cockpit»).

\section{Concept de valeur intrinsèque}

Les travaux du groupe de travail dédié aux valeurs intrinsèques, composé de 18 représentants des organisations faîtières de la $\mathrm{FMH}$, ont d'ores et déjà démarré. $\mathrm{Au}$ cours de cinq séances, le groupe de travail a développé trois nouveaux modèles visant à remplacer les valeurs intrinsèques quantitatives actuelles et défini des critères d'application pour les valeurs intrinsèques qualitatives. Après avoir adopté à l'unanimité les propositions élaborées, le groupe de travail les a présentées à l'Assemblée des délégués de la FMH le 26 janvier 2017. Les éléments des valeurs intrinsèques quantitatives, qui devront être approfondis ces prochains mois, peuvent être résumés comme suit:

Valeur intrinsèque individuelle propre au médecin (VIM): la valeur intrinsèque individuelle propre au médecin (VIM) doit permettre de rééquilibrer le revenu perçu tout au long de la vie active, qui peut différer selon le titre de spécialiste et la formation approfondie en raison des différentes durées de formation. Ce modèle tient uniquement compte du revenu issu d'activités figurant dans le tarif médical ambulatoire. A cet égard, les programmes de formation postgraduée de l'ISFM sont déterminants. La VIM est liée au médecin et peut être appliquée à la prestation médicale pour toutes les positions tarifaires.

Supplément pour les prestations au temps: avec les prestations à l'acte, des gains d'efficience sont en partie possibles lorsque le médecin a besoin de moins de temps pour fournir une prestation à un patient que la durée prévue par le tarif. Or le TARMED actuel (version 01.08.00_BR) ne permet pas aux spécialisations qui facturent principalement des prestations au temps d'en profiter: lorsque ces spécialistes traitent un patient de manière plus efficiente grâce à leur expérience, ils facturent alors une durée plus courte. Appliquer un supplément sur les prestations au temps à partir d'un certain degré d'expérience permettrait de remédier à cette situation. Ce supplément est lié au médecin et peut être appliqué à la prestation médicale pour toutes les positions tarifaires facturées au temps.

Facteur qualité lié à la prestation (FQP): le FQP est un instrument novateur visant à distribuer d'éventuels moyens financiers supplémentaires sur la base des critères de qualité définis par les sociétés de discipline médicale au sein des différents chapitres. Il se fonde sur l'ASQM et est lié à la prestation.

\section{Prochaines étapes}

Les collaborateurs du projet ont pris contact en début d'année avec les premières sociétés et ont commencé à planifier les premières séances des groupes de travail. Dès que les sociétés auront signé et retourné la convention de collaboration, les travaux pourront démarrer selon le calendrier ci-dessous.

Vous trouverez de plus amples informations sur le site internet de la FMH à l'adresse www.fmh.ch $\rightarrow$ Tarifs ambulatoires, et dans la lettre d'information du $\mathrm{Bu}$ reau des tarifs (www.fmh.ch $\rightarrow$ Tarifs ambulatoires $\rightarrow$ Publications $\rightarrow$ Lettre d'information).

\begin{tabular}{ll}
\hline Date & Etape \\
\hline 26.01 .2017 & Décision sur le concept détaillé \\
\hline $01.02 .2017-31.10 .2017$ & Travaux de fond au sein des groupes de travail \\
\hline $01.01 .2018-31.01 .2018$ & Phase de consultation (accent sur les valeurs intrinsèques qualitatives) \\
\hline 22.03 .2018 & Assemblée des délégués de la FMH: décision sur la nouvelle structure tarifaire \\
\hline 03.05 .2018 & $\begin{array}{l}\text { Chambre médicale de la FMH: décision sur la nouvelle structure tarifaire et, le cas échéant, } \\
\text { votation générale }\end{array}$ \\
\hline 30.06 .2018 & Remise des résultats du projet à l'Assemblée des délégués de la FMH \\
\hline 2018 & Finalisation avec les partenaires tarifaires et remise au Conseil fédéral \\
\hline
\end{tabular}

